\title{
DECOMPOSITION OF SUBSETS OF FINITE FIELDS
}

\author{
SIMON MACOURT
}

\begin{abstract}
We extend a bound of Roche-Newton, Shparlinski and Winterhof which says any subset of a finite field can be decomposed into two disjoint subset $\mathcal{U}$ and $\mathcal{V}$ of which the additive energy of $\mathcal{U}$ and $f(\mathcal{V})$ are small, for suitably chosen rational functions $f$. We extend the result by proving equivalent results over multiplicative energy and the additive and multiplicative energy hybrids.
\end{abstract}

\section{INTRODUCTION}

1.1. Background. Let $\mathbb{F}_{q}$ denote the finite field of $q$ elements of characteristic $p$.

Given two sets $\mathcal{U}, \mathcal{V} \subset \mathbb{F}_{q}$ we define their sum and product sets as

$$
\mathcal{U}+\mathcal{V}=\{u+v: u \in \mathcal{U}, v \in \mathcal{V}\} \text { and } \mathcal{U} \cdot \mathcal{V}=\{u v: u \in \mathcal{U}, v \in \mathcal{V}\}
$$

We define the additive and multiplicative energy of a set as follows

$$
\begin{aligned}
& E^{+}(\mathcal{U})=\#\left\{\left(u_{1}, u_{2}, u_{3}, u_{4}\right) \in \mathcal{U}^{4}: u_{1}+u_{2}=u_{3}+u_{4}\right\} \\
& E^{\times}(\mathcal{U})=\#\left\{\left(u_{1}, u_{2}, u_{3}, u_{4}\right) \in \mathcal{U}^{4}: u_{1} u_{2}=u_{3} u_{4}\right\} .
\end{aligned}
$$

We mention the sum-product problem which suggests that at least one of the sets $\mathcal{U}+\mathcal{V}$ and $\mathcal{U} \cdot \mathcal{V}$ must be large. This problem has been studied extensively in recent years, see [2]. There is a natural relation to the sum-product problem to bounds on additive and multiplicative energy. For example, by applying the Cauchy-Schwarz inequality one can see that

$$
E^{\times}(\mathcal{U}) \geqslant \frac{|\mathcal{U}|^{4}}{|\mathcal{U} \cdot \mathcal{U}|}
$$

and similarly for additive results. It follows that strong upper bounds on energy results correspond to strong lower bounds on the relevant sum-product estimate and vice-versa.

Balog and Wooley [1] proved that in finite fields the set $\mathcal{U}$ can be decomposed into a disjoint union of subsets $\mathcal{V}$ and $\mathcal{W}$ such that $E^{+}(\mathcal{V})$ and $E^{\times}(\mathcal{W})$ are both small. These results have been improved on by Konyagin and Shkredov [3] and Rudnev, Shkredov and Stevens [5].

Our main results are an extension of [4], which themselves are a generalisation of the Balog-Wooley decomposition [1, Theorem 1.3]. 
Here we extend the results of [4] to multiplicative energy and the hybrid cases of both additive and multiplicative energy.

1.2. Notation. For $a \in \mathbb{F}_{q}$ and a rational function $f \in \mathbb{F}_{q}(X)$ we use $r_{\mathcal{U}, \mathcal{V}}^{+}(f, a)$ to denote the number of solutions to $f(u)+f(v)=a,(u, v) \in$ $\mathcal{U} \times \mathcal{V}$. Similarly we use $r_{\mathcal{U}, \mathcal{V}}^{\times}(f, a)$ to denote the number of solutions to $f(u) f(v)=a$. If $\mathcal{U}=\mathcal{V}$ we write $r_{\mathcal{U}}^{+}(f, a)$ and if $f(X)=X$ we write $r_{\mathcal{U}, \mathcal{V}}^{+}(a)$.

For this paper we use the convention that capital letters in italics, such as $\mathcal{U}$, will be used to represent sets. Corresponding capital letters in roman will denote their cardinalities, such as $U=|\mathcal{U}|$. We also use $\mathcal{X}$ and $\Psi^{*}$ to denote the sets of additive and multiplicative characters respectively, and we will use the lower case $\chi$ and $\psi$ to represent their respective characters.

Throughout the paper we use the notation $A \ll B$ to indicate $|A| \leqslant c|B|$ for some absolute constant $c$. We also use the notation $A \ll_{k} B$ for when the constant $c$ depends on some parameter $k$. We also equivalently write $A=O(B)$ and $A=O_{k}(B)$.

1.3. Main Results. Here we extend the result of [4, Theorem 1.1] to multiplicative energy and a hybrid of additive and multiplicative energies.

Theorem 1.1. For any set $\mathcal{A} \subset \mathbb{F}_{q}^{*}$ and any rational function $f \in \mathbb{F}_{q}(X)$ of degree $k$ which is not of the form $f(X)=\operatorname{rg}(X)^{d} X^{\lambda}$ where $d \mid q-1$, there exist disjoint sets $\mathcal{S}, \mathcal{T} \subset \mathcal{A}$ such that $\mathcal{A}=\mathcal{S} \cup \mathcal{T}$ and

$$
\max \left\{E^{\times}(\mathcal{S}), E^{\times}(f(\mathcal{T}))\right\} \ll_{k} \frac{A^{3}}{M(A)}
$$

where

$$
M(\mathcal{A})=\min \left\{\frac{q^{1 / 2}}{A^{1 / 2}(\log A)^{11 / 4}}, \frac{A^{4 / 5}}{q^{2 / 5}(\log A)^{31 / 10}}\right\} .
$$

Theorem 1.2. For any set $\mathcal{A} \subset \mathbb{F}_{q}^{*}$ and any rational function $f \in \mathbb{F}_{q}(X)$ of degree $k$ which is not of the form $f(X)=g\left(X^{p}\right)-g(X)+\lambda X+\mu$, there exist disjoint sets $\mathcal{S}, \mathcal{T} \subset \mathcal{A}$ such that $\mathcal{A}=\mathcal{S} \cup \mathcal{T}$ and

$$
\max \left\{E^{\times}(\mathcal{S}), E^{+}(f(\mathcal{T}))\right\} \ll_{k} \frac{A^{3}}{M(A)} .
$$

Theorem 1.3. For any set $\mathcal{A} \subset \mathbb{F}_{q}^{*}$ and any rational function $f \in \mathbb{F}_{q}(X)$ of degree $k$ which is not of the form $f(X)=r g(X)^{d} X^{\lambda}$ where $d \mid q-1$, 
there exist disjoint sets $\mathcal{S}, \mathcal{T} \subset \mathcal{A}$ such that $\mathcal{A}=\mathcal{S} \cup \mathcal{T}$ and

$$
\max \left\{E^{+}(\mathcal{S}), E^{\times}(f(\mathcal{T}))\right\} \ll_{k} \frac{A^{3}}{M(A)} .
$$

\section{Sum-Product}

2.1. Preliminary Results. We give a series of lemmas, the proofs of which follow those of [4] with multiplicative characters replacing additive characters and other equivalent substitutions.

Lemma 2.1. Let $(\chi, \psi) \in \mathcal{X} \times \Psi^{*}$. For any rational function $f \in \mathbb{F}_{q}(X)$ of degree $k$, if $\chi$ is non-trivial, and not of the form $f(X)=r g(X)^{d} X^{\lambda}$ where $d$ is the order of $\chi$, if $\psi$ is trivial, we have

$$
\sum_{u \in \mathcal{U}} \sum_{v \in \mathcal{V}} \chi(f(u v)) \psi(u v) \ll_{k} \sqrt{U V q}
$$

Proof. Let

$$
\Sigma=\sum_{u \in \mathcal{U}} \sum_{v \in \mathcal{V}} \chi(f(u v)) \psi(u v)
$$

Then,

$$
\begin{aligned}
\Sigma & =\sum_{x \in \mathbb{F}_{q}} \psi(x) \chi(f(x)) \frac{1}{q-1} \sum_{\lambda=1}^{q-1} \sum_{u \in \mathcal{U}} \sum_{v \in \mathcal{V}} \chi\left(\left(u v x^{-1}\right)^{\lambda}\right) \\
& =\frac{1}{q-1} \sum_{\lambda=1}^{q-1} \sum_{x \in \mathbb{F}_{q}} \psi(x) \chi\left(f(x)\left(x^{-1}\right)^{\lambda}\right) \sum_{u \in \mathcal{U}} \chi\left(u^{\lambda}\right) \sum_{v \in \mathcal{V}} \chi\left(v^{\lambda}\right) .
\end{aligned}
$$

By the Weil bound we have

$$
\Sigma \ll q^{-1 / 2} \sum_{\lambda=1}^{q-1}\left|\sum_{u \in \mathcal{U}} \chi\left(u^{\lambda}\right)\right|\left|\sum_{v \in \mathcal{V}} \chi\left(v^{\lambda}\right)\right| .
$$

Using the Cauchy-Schwarz inequality we obtain

$$
\begin{aligned}
\sum_{\lambda \in \mathbb{F}_{q}}\left|\sum_{u \in \mathcal{U}} \chi\left(u^{\lambda}\right)\right| \mid & \sum_{v \in \mathcal{V}} \chi\left(v^{\lambda}\right) \mid \\
& \leqslant\left(\sum_{\lambda \in \mathbb{F}_{q}}\left|\sum_{u \in \mathcal{U}} \chi\left(u^{\lambda}\right)\right|^{2}\right)^{1 / 2}\left(\sum_{\lambda \in \mathbb{F}_{q}}\left|\sum_{v \in \mathcal{V}} \chi\left(v^{\lambda}\right)\right|^{2}\right)^{1 / 2} \\
& \leqslant\left(q^{2} U V\right)^{1 / 2} .
\end{aligned}
$$


Lemma 2.2. Suppose $\mathcal{U}, \mathcal{V}, \mathcal{Y}, \mathcal{Z} \subset \mathbb{F}_{q}$. For any rational function $f \in$ $\mathbb{F}_{q}(X)$ of degree $k$ which is not of the form $f(X)=\operatorname{rg}(X)^{d} X^{\lambda}$ where $d \mid q-1$ and $d \geqslant 2$, the number of solutions $J$ to the equation

$$
f(u v)=y z \quad(u, v, y, z) \in \mathcal{U} \times \mathcal{V} \times \mathcal{Y} \times \mathcal{Z}
$$

satisfies the bound

$$
J \leqslant \frac{U V Y Z}{q-1}+O_{k}\left((U V Y Z q)^{1 / 2}\right)
$$

Proof. Using the approximate orthogonality of multiplicative characters, we have

$$
J \leqslant \sum_{(u, v, y, z) \in \mathcal{U} \times \mathcal{V} \times \mathcal{Y} \times \mathcal{Z}} \frac{1}{q-1} \sum_{\chi \in \mathcal{X}} \chi\left(f(u v)(y z)^{-1}\right) .
$$

Re-arranging and separating the contribution from the trivial character

$$
J-\frac{U V Y Z}{q-1} \leqslant \frac{1}{q-1} \sum_{\chi \in \mathcal{X}^{*}}\left|\sum_{(u, v) \in \mathcal{U} \times \mathcal{V}} \chi(f(u v))\right|\left|\sum_{y \in \mathcal{Y}} \chi\left(y^{-1}\right)\right|\left|\sum_{z \in \mathcal{Z}} \chi\left(z^{-1}\right)\right| .
$$

Now by Lemma 2.1 with the trivial additive character, we have

$$
\begin{aligned}
J-\frac{U V Y Z}{q-1} & \ll_{k} \frac{\sqrt{U V q}}{q-1} \sum_{\chi \in \mathcal{X}^{*}}\left|\sum_{y \in \mathcal{Y}} \chi\left(y^{-1}\right)\right|\left|\sum_{z \in \mathcal{Z}} \chi\left(z^{-1}\right)\right| \\
& \ll_{k} \frac{\sqrt{U V}}{q^{1 / 2}} \cdot\left(q^{2} Y Z\right)^{1 / 2} .
\end{aligned}
$$

This completes the proof.

Lemma 2.3. Let $\mathcal{A}, \mathcal{S}, \mathcal{U} \subset \mathbb{F}_{q}^{*}$. Let $u>0$ be such that $r_{\mathcal{S}, \mathcal{A}^{-1}}^{\times}(x) \geqslant u$ for all $x \in \mathcal{U}$. Let $k$ be a fixed positive integer and suppose also that

$$
\tau \geqslant 2 \frac{k A S U}{u q}
$$

Then, for any rational function $f \in \mathbb{F}_{q}(X)$ of degree $k$ which is not of the form $f(X)=r g(X)^{d} X^{\lambda}$ where $d \mid p-1$ and $d \geqslant 2$, we have

$$
\#\left\{x \in \mathbb{F}_{q}: r_{\mathcal{U}}^{\times}(f, x) \geqslant \tau\right\} \ll_{k} \frac{A U S q}{u^{2} \tau^{2}} .
$$

Proof. Our proof follows [4, Lemma 2.3] where here we replace $r_{\mathcal{U}}(f, x)$ with $r_{\mathcal{U}}^{\times}(f, x)$. Define

$$
\mathcal{R}=\left\{x \in \mathbb{F}_{q}: r_{\mathcal{U}}^{\times}(f, x) \geqslant \tau\right\} .
$$


Clearly,

$$
\tau R \leqslant \sum_{x \in \mathcal{R}} r_{\mathcal{U}}(f, x)=\{(x, y, z) \in \mathcal{R} \times \mathcal{U} \times \mathcal{U}: x=f(y) f(z)\}
$$

Now $r_{\mathcal{S}, \mathcal{A}^{-1}}(z) \geqslant u$ for $z \in \mathcal{U}$, hence

$$
\begin{aligned}
& \#\{(x, y, z) \in \mathcal{R} \times \mathcal{U} \times \mathcal{U}: x=f(y)+f(z)\} \\
& \leqslant u^{-1} \#\left\{(v, w, x, y) \in \mathcal{S} \times \mathcal{A} \times \mathcal{R} \times \mathcal{U}: x=f(y) f\left(v w^{-1}\right)\right\} .
\end{aligned}
$$

Therefore, we have

$$
\begin{aligned}
\tau U R & \leqslant \#\left\{(v, w, x, y) \in \mathcal{S} \times \mathcal{A} \times \mathcal{R} \times \mathcal{U}: x=f(y) f\left(v w^{-1}\right)\right\} \\
& \leqslant k \cdot \#\left\{(v, w, x, z) \in \mathcal{S} \times \mathcal{A} \times \mathcal{R} \times f(\mathcal{U}): x=z f\left(v w^{-1}\right)\right\} .
\end{aligned}
$$

We then apply Lemma 2.2 to obtain

$$
\tau U R \leqslant \frac{k A R S U}{q}+O_{k}\left((A R S U q)^{1 / 2}\right)
$$

The assumed lower bound on $\tau$ implies

$$
\tau U R \ll_{k}(A R S U q)^{1 / 2}
$$

This concludes the proof.

Lemma 2.4. Let $\mathcal{A}_{1}, \ldots, \mathcal{A}_{n} \subset \mathbb{F}_{q}^{*}$. Then

$$
E^{\times}\left(\bigcup_{i=1}^{n} \mathcal{A}_{i}\right) \leqslant\left(\sum_{n=1}^{n} E^{\times}\left(\mathcal{A}_{i}\right)^{1 / 4}\right)^{4}
$$


Proof. We assume the sets $\mathcal{A}_{i}, \ldots, \mathcal{A}_{n}$ are disjoint. Then using the Cauchy-Schwarz inequality twice we have,

$$
\begin{aligned}
E^{\times}\left(\bigcup_{i=1}^{n} \mathcal{A}_{i}\right) & =\sum_{i, j, k, \ell=1}^{n} \sum_{x \in \mathbb{F}_{q}} r_{\mathcal{A}_{i}, \mathcal{A}_{j}}^{\times}(x) r_{\mathcal{A}_{k}, \mathcal{A}_{\ell}}^{\times}(x) \\
& \leqslant \sum_{i, j, k, \ell=1}^{n}\left(\sum_{x \in \mathbb{F}_{q}} r_{\mathcal{A}_{i}, \mathcal{A}_{j}}^{\times}(x)^{2}\right)^{1 / 2}\left(\sum_{x \in \mathbb{F}_{q}} r_{\mathcal{A}_{k}, \mathcal{A}_{\ell}}^{\times}(x)^{2}\right)^{1 / 2} \\
& =\left(\sum_{i, j=1}^{n}\left(\sum_{x \in \mathbb{F}_{q}} r_{\mathcal{A}_{i}, \mathcal{A}_{j}}^{\times}(x)^{2}\right)^{1 / 2}\right)^{2} \\
& \left.\left.=\left(\sum_{i, j=1}^{n}\left(\sum_{x \in \mathbb{F}_{q}} r_{\mathcal{A}_{i}, \mathcal{A}_{i}^{-1}}^{\times}(x) r_{\mathcal{A}_{j}, \mathcal{A}_{j}^{-1}}^{\times}(x)\right)^{1 / 2}\right)^{2}\right)^{1 / 4}\left(\sum_{x \in \mathbb{F}_{q}} r_{\mathcal{A}_{j}, \mathcal{A}_{j}^{-1}}^{\times}(x)^{2}\right)^{1 / 4}\right)^{2} \\
& \leqslant\left(\sum_{i, j=1}^{n}\left(\sum_{x \in \mathbb{F}_{q}} r_{\mathcal{A}_{i}, \mathcal{A}_{i}^{-1}}^{\times}(x)^{2}\right)^{4}\right. \\
& =\left(\sum_{i=1}^{n}\left(\sum_{x \in \mathbb{F}_{q}} r_{\mathcal{A}_{i}, \mathcal{A}_{i}^{-1}}^{\times}(x)^{2}\right)^{1 / 4}\right)^{4}\left(\sum_{i=1}^{n} E^{\times}\left(\mathcal{A}_{i}\right)^{1 / 4}\right)^{4}
\end{aligned}
$$

This concludes the proof.

Lemma 2.5. Let $\mathcal{A} \subset \mathbb{F}_{q}$. Then for any rational function $f \in \mathbb{F}_{q}(X)$ of degree $k$ which is not of the form $f(X)=\operatorname{rg}(X)^{d} X^{\lambda}$ where $d \mid p-1$ and $d \geqslant 2$, there exists $\mathcal{U} \subset \mathcal{A}$ of cardinality $U$ such that

$$
U \gg \frac{E^{\times}(\mathcal{A})^{1 / 2}}{A^{1 / 2}(\log A)^{7 / 4}}
$$

and

$$
E^{\times}(f(\mathcal{U})) \ll_{k} \frac{A U^{6} q^{-1}(\log A)^{11 / 2}+A U^{3} q(\log A)^{6}}{E^{\times}(\mathcal{A})} .
$$

Proof. Clearly,

$$
E^{\times}(\mathcal{A})=\sum_{x \in \mathcal{A} \cdot \mathcal{A}} r_{\mathcal{A}}^{\times}(x)^{2}
$$

We dyadically decompose this sum and define the set

$$
\mathcal{S}^{\times}=\left\{x \in \mathcal{A} \cdot \mathcal{A}: \rho \leqslant r_{\mathcal{A}}^{\times}(x)<2 \rho\right\}
$$


with some integer $1 \leqslant \rho \leqslant A$ where $\rho$ is a power of 2 , and such that

$$
\rho^{2} S \gg \frac{E^{\times}(\mathcal{A})}{\log A} .
$$

Consider

$$
\mathcal{P}=\left\{(a, b) \in \mathcal{A} \times \mathcal{A}: a b \in \mathcal{S}^{\times}\right\}
$$

Now we have

$$
\rho S \leqslant P<2 \rho S .
$$

We then make another dyadic decomposition of $\mathcal{S}$ to find a large subset supported on vertical lines. That is, we define

$$
\mathcal{A}_{x}=\{y:(x, y) \in \mathcal{P}\} .
$$

Therefore, for some $s$ there exists a dyadic set

$$
\mathcal{V}=\left\{x \in \mathcal{A}: s \leqslant \mathcal{A}_{x}<2 s\right\}
$$

such that

$$
V s \gg \frac{P}{\log A} \gg \frac{\rho S}{\log A} .
$$

We now separate into two cases. First, suppose

$$
V \geqslant \frac{s}{(\log A)^{1 / 2}} \text {. }
$$

Then for any $x \in \mathcal{V}$, there exist

$$
y_{1}, y_{2}, \ldots, y_{s} \in \mathcal{A}_{x} \subset \mathcal{A}
$$

such that $\left(x, y_{i}\right) \in \mathcal{P}$ for all $1 \leqslant i \leqslant s$. Therefore

$$
x y_{1}, x y_{2}, \ldots, x y_{s} \in \mathcal{S}^{\times} .
$$

It follows that $r_{\mathcal{S}^{\times}, \mathcal{A}^{-1}}^{\times}(x) \geqslant s$ for every $x \in \mathcal{V}$ and in this case we define

$$
\mathcal{U}=\mathcal{V} \quad \text { and } \quad u=s .
$$

Now suppose

$$
V<\frac{s}{(\log A)^{1 / 2}}
$$

We now consider the point set

$$
\mathcal{Q}=\{(x, y) \in \mathcal{P}: x \in \mathcal{V}\} .
$$

As before, for any $x \in \mathcal{V}$ there exist at least $s$ values of $y \in \mathcal{A}_{x} \subset \mathcal{A}$ with $(x, y) \in \mathcal{P}$. Hence $Q \geqslant V s$. 
For any $y \in \mathbb{F}_{q}$ we define

$$
\mathcal{B}_{y}=\{x:(x, y) \in \mathcal{Q} .
$$

Clearly,

$$
\sum_{y \in \mathcal{A}} B_{y}=Q
$$

Therefore, for some $t$ there exists a dyadic set

$$
\mathcal{W}=\left\{y \in \mathcal{A}: t \leqslant B_{y}<2 t\right\}
$$

such that

$$
W t \gg \frac{Q}{\log A} \geqslant \frac{V s}{\log A} .
$$

Now since $\mathcal{Q} \subset \mathcal{V} \times \mathcal{A}$ we also have $t \leqslant V$. From (2.5) and our assumption on $s$ we have

$$
W V \geqslant W t \gg \frac{V s}{\log A}>\frac{V^{2}}{(\log A)^{1 / 2}}
$$

hence

$$
W \gg \frac{V}{(\log A)^{1 / 2}} \geqslant \frac{t}{(\log A)^{1 / 2}} .
$$

Now, by (2.5) and (2.3)

$$
W t \gg \frac{V s}{\log A} \gg \frac{\rho S}{(\log A)^{2}} .
$$

Now, let $y \in \mathcal{W}$. Then there exist $x_{1}, \ldots, x_{t} \in \mathcal{A}$ such that $\left(x_{i}, y\right) \in \mathcal{P}$ for all $1 \leqslant i \leqslant t$. Therefore,

$$
x_{1} y, \ldots, x_{t} y \in \mathcal{S}
$$

Then $r_{\mathcal{S}, \mathcal{A}^{-1}}^{\times}(y) \geqslant t$ for every $y \in \mathcal{W}$.

We then take

$$
\mathcal{U}=\mathcal{W} \quad \text { and } \quad u=t .
$$

It is clear for both (2.4) and (2.8) we have $\mathcal{U} \subset \mathcal{A}$,

$$
U \gg \frac{u}{(\log A)^{1 / 2}}
$$

and

$$
u U \gg \frac{\rho S}{(\log A)^{2}}
$$


where $r_{\mathcal{S}, \mathcal{A}^{-1}}^{\times}(x) \geqslant u$ for all $x \in \mathcal{U}$. Multiplying (2.9) and (2.10) and using (2.1) we obtain

$$
U^{2} \gg \frac{\rho S}{(\log A)^{5 / 2}} \gg \frac{E^{\times}(\mathcal{A})}{A(\log A)^{7 / 2}} .
$$

We now need a bound on $E^{\times}(f(\mathcal{U}))$. We have

$$
E^{\times}(f(\mathcal{U}))=\sum_{x \in \mathbb{F}_{q}} r_{f(\mathcal{U})}^{\times}(x)^{2} \leqslant \sum_{x \in \mathbb{F}_{q}} r_{\mathcal{U}}^{\times}(f, x)^{2} .
$$

We define the set

$$
\mathcal{R}_{0}=\left\{x \in \mathbb{F}_{q}: r_{\mathcal{U}}^{\times}(f, x) \leqslant 2 \frac{k A S U}{u q}\right\}
$$

and for $J=\lceil\log A / \log 2\rceil$, we define the sets

$$
\mathcal{R}_{j}=\left\{x \in \mathbb{F}_{q}: 2^{j} \frac{k A S U}{u q}<r_{\mathcal{U}}^{\times}(f, x) \leqslant 2^{j+1} \frac{A k S U}{u q}\right\}, j=1, \ldots, J .
$$

Since,

$$
\sum_{x \in \mathbb{F}_{q}} r_{\mathcal{U}}^{\times}(f, x)=U^{2}
$$

we have

$$
\sum_{x \in \mathbb{R}_{0}} r_{\mathcal{U}}^{\times}(f, x)^{2} \leqslant 2 \frac{k A S U}{u q} \sum_{x \in \mathbb{F}_{q}} r_{\mathcal{U}}^{\times}(f, x) \ll \frac{k A S U^{3}}{u q} .
$$

For $i=1, \ldots, J$, we apply Lemma 2.3 with

$$
\tau=2^{j} \frac{A k S U}{u q}
$$

to obtain

$$
\sum_{x \in \mathbb{R}_{j}} r_{\mathcal{U}}^{\times}(f, x)^{2} \leqslant(2 \tau)^{2} R_{j} \ll_{k} \frac{A S U q}{u^{2}} .
$$

Combining (2.13) and (2.14) we get

$$
E^{\times}(f(\mathcal{U})) \ll_{k} \frac{A S U^{3}}{u q}+\frac{A S U q}{u^{2}} \log A .
$$

Now, multiplying (2.10) with (2.11) and applying (2.1), we obtain

which gives

$$
u U^{3} \gg \frac{\rho^{2} S^{2}}{(\log A)^{9 / 2}} \gg \frac{S E^{\times}(\mathcal{A})}{(\log A)^{11 / 2}}
$$

$$
\frac{S}{u} \ll \frac{U^{3}(\log A)^{11 / 2}}{E^{\times}(\mathcal{A})}
$$


Also, squaring (2.10) and applying (2.1)

$$
u^{2} U^{2} \gg \frac{\rho^{2} S^{2}}{(\log A)^{4}} \gg \frac{S E^{\times}(\mathcal{A})}{(\log A)^{5}}
$$

which gives

$$
\frac{S}{u^{2}} \ll \frac{U^{2}(\log A)^{5}}{E^{\times}(\mathcal{A})} .
$$

Applying (2.16) and (2.17) into the first and second terms of (2.15) respectively we obtain

$$
E^{\times}(f(\mathcal{U})) \ll_{k} \frac{A U^{6} q^{-1}(\log A)^{11 / 2}+A U^{3} q(\log A)^{6}}{E^{\times}(\mathcal{A})} .
$$

This concludes the proof.

Corollary 2.6. Let $\mathcal{A} \subset \mathbb{F}_{q}$. Then for any rational function $f \in \mathbb{F}_{q}(X)$ of degree $k$ which is not of the form $f(X)=g\left(X^{p}\right)-g(X)+\lambda X+\mu$, there exists $\mathcal{U} \subset \mathcal{A}$ of cardinality $U$ such that

$$
U \gg \frac{E^{\times}(\mathcal{A})^{1 / 2}}{A^{1 / 2}(\log A)^{7 / 4}}
$$

and

$$
E^{+}(f(\mathcal{U})) \ll_{k} \frac{A U^{6} q^{-1}(\log A)^{11 / 2}+A U^{3} q(\log A)^{6}}{E^{\times}(\mathcal{A})} .
$$

Proof. We follow the proof of Lemma 2.5, however we replace $E^{\times}$with $E^{+}$in (2.12), and then use analogous results following from [4, Equation 2.12].

Corollary 2.7. Let $\mathcal{A} \subset \mathbb{F}_{q}$. Then for any rational function $f \in \mathbb{F}_{q}(X)$ of degree $k$ which is not of the form $f(X)=\operatorname{rg}(x)^{d} x^{\lambda}$ where $d \mid p-1$ and $d \geqslant 2$, there exists $\mathcal{U} \subset \mathcal{A}$ of cardinality $U$ such that

$$
U \gg \frac{E^{+}(\mathcal{A})^{1 / 2}}{A^{1 / 2}(\log A)^{7 / 4}}
$$

and

$$
E^{\times}(f(\mathcal{U})) \ll_{k} \frac{A U^{6} q^{-1}(\log A)^{11 / 2}+A U^{3} q(\log A)^{6}}{E^{+}(\mathcal{A})} .
$$

Proof. We follow the proof of [4, Lemma 2.5], however we replace $E^{+}$ with $E^{\times}$in equation $(2.12)$ and the proceed as in our Lemma 2.5. 


\subsection{Proofs of Theorems 1.1, 1.2 and $\mathbf{1 . 3 .}$}

Proof. The proofs follow that of [4, Theorem 1.1], but for Theorem 1.1 our new multiplicative results from Lemma 2.5 are used in place of additive results. For Theorems 1.2 and 1.3 our mixed results from Corollaries 2.6 and 2.7 are used respectively.

\section{REFERENCES}

[1] A. Balog and T. Wooley, A low-energy decomposition theorem, Q. J. Math. 68 (2017), no. 1, 207-226.

[2] J. Bourgain, N. Katz, and T. Tao, A sum-product estimate in finite fields, and applications, Geom. Funct. Anal. 14 (2004), no. 1, 27-57.

[3] S. V. Konyagin and I. D. Shkredov, New results on sums and products in $\mathbb{R}$, Tr. Mat. Inst. Steklova 294 (2016), no. Sovremennye Problemy Matematiki, Mekhaniki i Matematicheskoŭ Fiziki. II, 87-98.

[4] O. Roche-Newton, I. Shparlinski, and A. Winterhof, Analogues of the BalogWooley Decomposition for Subsets of Finite Fields and Character Sums with Convolutions, Ann. Comb. (to appear).

[5] M. Rudnev, I. D. Shkredov, and S. Stevens, On The Energy Variant of the Sum-Product Conjecture, ArXiv e-prints (2016).

Department of Pure Mathematics, University of New South Wales, Sydney, NSW 2052, Australia

E-mail address: s.macourt@student.unsw.edu.au 